\title{
A Percepção de Justiça como Antecedente do Compro- metimento Organizacional: um Estudo Luso-Brasileiro
}

\author{
Arménio Rego \\ Solange Souto
}

\begin{abstract}
Resumo
O estudo visa mostrar como as percepções de justiça explicam o comprometimento organizacional (afetivo, normativo e instrumental). Inquiriram-se 229 membros de organizações brasileiras e 236 de organizações portuguesas. Os dados sugerem o seguinte: (1) o modelo de quatro dimensões de justiça (distributiva, procedimental, interpessoal e informacional) denota valia psicométrica superior ao de três dimensões, no qual as vertentes interpessoal e informacional são agrupadas numa mesma dimensão interaccional; (2) as percepções de justiça explicam entre 23\% (Portugal) e 28\% (Brasil) do comprometimento afetivo, entre 15\% (Brasil) e 37\% (Portugal) do normativo, e entre 1\% (Brasil) e 6\% (Portugal) do instrumental; (3) as vertentes de justiça mais pertinentes para a explicação do laço afetivo são a procedimental e a interpessoal; (4) o laço normativo tende a ser explicado pelas facetas distributiva, procedimental e informacional da justiça; (5) globalmente, quando se sentem tratadas com justiça, as pessoas tendem a denotar mais fortes laços afetivos e normativos, e menores índices de comprometimento instrumental; (6) estas tendências são, em geral, aplicáveis às duas amostras; (7) a compreensão do comprometimento organizacional é mais proveitosa quando se estudam os diferentes modos como as pessoas combinam os laços afetivo, normativo e instrumental. O estudo ajuda a compreender as razões pelas quais as pessoas se comprometem psicologicamente com as suas organizações. Seu valor torna-se maior pelo fato de combinar dados de duas culturas diferentes.
\end{abstract}

Palavras-chaves: comprometimento afetivo; comprometimento normativo; comprometimento instrumental; justiça organizacional.

\begin{abstract}
The study aims at showing how perceptions of justice predict organizational commitment (affective, normative, continuance). Two samples comprising 229 individuals from Brazilian organizations and 236 from Portuguese organizations were collected. The data suggest the following: (1) the model of four dimensions of justice (distributive, procedural, interpersonal, informational) shows better psychometric properties than the three-factor model (in which the interpersonal and informational dimensions were aggregated in the interactional factor); (2) the justice perceptions explain between 23\% (Portugal) and 28\% (Brasil) of the variance of affective commitment, between 15\% (Brazil) and 37\% (Portugal) of the variance of the normative commitment, and between 1\% (Brazil) and 6\% (Portugal) of the variance of the continuance commitment; (3) the justice dimensions with higher predictive value for commitment are the procedural and the interpersonal; (4) normative dimension is explained by distributive, procedural and informational justice; (5) in general, people that feels fairly treated tends to denote stronger affective and normative bonds with the organization, and weaker continuance bonds; (6) these tendencies are common to the Brazilian and Portuguese samples; (7) organizational commitment can be better understood when the different bonds are combined in specific patterns. The study contributes to a better understanding of the motives by which people feels committed to their organizations. Its value is still more relevant due to the fact it combines data from two different cultures.
\end{abstract}

Key words: affective commitment; normative commitment; continuance commitment; organizational justice. 


\section{INTRODUÇÃO}

\section{Linhas-Mestras do Artigo}

O comprometimento organizacional é uma das variáveis que mais dedicação tem recebido da parte dos investigadores em psicologia organizacional (Allen e Meyer, 2000; Swailes, 2000) Tal facto radica porventura na premissa de que desta atitude decorrem efeitos substanciais para o desempenho dos indivíduos e das organizações. Por conseguinte, têm sido numerosas as tentativas destinadas a pesquisar os seus antecedentes (causas). É neste domínio que se insere o presente artigo. É seu objectivo testar em que grau as percepções de justiça explicam três componentes do comprometimento: afectivo, normativo e instrumental. Visa, também, cotejar as propriedades psicométricas de dois modelos de justiça. O primeiro integra três dimensões (distributiva, procedimental e interaccional). O segundo, que abarca quatro dimensões, resulta da partição da dimensão interaccional em dois factores: interpessoal e informacional. O texto está assim organizado:

. Começamos por referir a relevância que o comprometimento tem assumido nas investigações em psicologia organizacional.

. Abordamos então a multidimensionalidade do construto.

- Referimos depois a justiça como um dos potenciais antecedentes do comprometimento, fazendo também menção à sua multidimensionalidade.

. Expomos o método usado para realizar a pesquisa empírica.

. Depois de exibirmos os resultados, analisamo-los e discutimo-los. Redigimos então algumas conclusões e fazemos luz sobre algumas limitações da pesquisa.

\section{Comprometimento Organizacional e Desempenho Organizacional}

Envolvidas em ambientes (tecnológicos, de mercado, laborais) progressivamente turbulentos, inseridas em contextos competitivos à escala global, enfrentando problemas inéditos reivindicando soluções criativas/ 
inovadoras, as organizações necessitam cada vez mais do comprometimento dos seus colaboradores. Enunciando de outro modo: precisam que os seus membros denotem amor à camisa, espírito de entrega, orientação para elevados desempenhos e disponibilidade para executar comportamentos de cidadania (Rego, 2002).

Na senda desses propósitos, os gestores tentam desenvolver ações e políticas visando promover os vínculos psicológicos (entre os indivíduos e as organizações) que favoreçam a competitividade organizacional. Os pesquisadores têm correspondido a tais intentos, propondo diversos construtos que representam tais vínculos. Os contratos psicológicos (Rousseau, 1995; Morrison e Robinson, 1997), o sentido psicológico de comunidade de trabalho (Burroughs e Eby, 1998), a identificação organizacional (Mael e Ashforth, 1992; Dutton, Duberich e Harquail, 1994; Van Knippenberg e Van Schie, 2000) e o comprometimento organizacional (por exemplo, Allen e Meyer, 1990, 1993, 1996; Meyer e Allen, 1991; Meyer, 1997) representam, porventura, os exemplos mais eloqüentes dessa convergência de preocupações entre investigadores e gestores.

O enfoque deste artigo é o comprometimento organizacional. Numa enunciação simples, ele pode ser concebido como o laço psicológico que caracteriza a ligação dos indivíduos à organização, e que tem implicações para a decisão de nela permanecerem (Meyer e Allen, 1991; Allen e Meyer, 1996; Meyer, 1997). Desde há várias décadas que o tópico vem ocupando espaço notório entre as preocupações dos investigadores em psicologia industrial/organizacional - fato que induziu Swailes (2000, p.199, sem grifo no original) a uma enunciação bastante peremptória: "será difícil encontrar na literatura sobre organizações um tópico que tenha atraído mais interesse do que o empenhamento".

Este marcante interesse reside, de modo considerável, na evidência de que o comprometimento pode ter conseqüências em várias atitudes e comportamentos organizacionalmente relevantes, sendo de destacar as seguintes (O' Reilly e Chatman, 1986; Meyer et al., 1989; Konovsky e Cropanzano, 1991; Becker, 1992; Meyer, Allen e Smith, 1993; Hackett, Bycio e Hausdorf, 1994; Allen e Meyer, 1996; Bolon, 1997; Kim e Mauborgne, 1997; Medeiros, 1997; Meyer, 1997; MacKenzie, Podsakoff e Ahearne, 1998; Caetano e Vala, 1999; Iverson e Buttigieg, 1999; Bishop, Scott e Burroughs, 2000; Clugston, 2000; Cohen, 2000; Rego, 2002; Yousef, 2000): assiduidade, intenções de abandonar a organização, abandono efetivo, pontualidade, atitudes favoráveis face à mudança, desempenho individual, comportamentos de cidadania, comportamentos de negligência, capital humano, desempenho organizacional. Goleman (1999) testemunhou exuberantemente a importância do tema ao aduzir que as pessoas comprometidas tendem a atuar como 'patriotas' das suas organizações e 


\section{representam modelos de atuação que os restantes membros organizacionais tendem a imitar.}

\section{A Multidimensionalidade do Comprometimento ORGANIZACIONAL}

A interpretação das conseqüências e dos antecedentes do comprometimento só pode ser profícua após a compreensão da sua multidimensionalidade. $\mathrm{Na}$ verdade, os estudos têm revelado que diferentes dimensões são explicadas por diferentes antecedentes e explicam distintas consequências. Embora alguns autores advoguem uma estrutura tetra-dimensional (por exemplo, McGee e Ford, 1987; Meyer e Allen, 1991; Hackett, Bycio e Hausdorf, 1994; Allen e Meyer, 1996; Iverson e Buttigieg, 1999), a tese mais comum propõe as três dimensões que o Quadro 1 expõe.

\section{Quadro 1: As Três Componentes Mais Comuns do Comprometimento Organizacional}

\begin{tabular}{|c|c|c|c|}
\hline Categorias & Caracterização & $\begin{array}{l}\text { A pessoa } \\
\text { permanece na } \\
\text { organização } \\
\text { porque ... }\end{array}$ & $\begin{array}{l}\text { Estado } \\
\text { psicológico }\end{array}$ \\
\hline Afetivo & $\begin{array}{l}\text { Grau em que o colaborador se sente emocionalmente ligado, } \\
\text { identificado e envolvido na organização. }\end{array}$ & $\begin{array}{l}\text {... sente que quer } \\
\text { permanecer. }\end{array}$ & Desejo \\
\hline Normativo & $\begin{array}{l}\text { Grau em que o colaborador possui um sentido da obrigação (ou } \\
\text { dever moral) de permanecer na organização. }\end{array}$ & $\begin{array}{l}\text {... sente que deve } \\
\text { permanecer. }\end{array}$ & Obrigação \\
\hline Instrumental & $\begin{array}{l}\text { Grau em que o colaborador se mantém ligado à organização devido } \\
\text { ao reconhecimento dos custos associados com a sua saída dela. } \\
\text { Este reconhecimento pode advir da ausência de alternativas de } \\
\text { emprego, ou do sentimento de que os sacrifícios pessoais gerados } \\
\text { pela saída serão elevados. }\end{array}$ & $\begin{array}{l}\text {... sente que tem } \\
\text { necessidade de } \\
\text { permanecer. }\end{array}$ & Necessidade \\
\hline
\end{tabular}

Fonte: Rego (2003) com base em Allen e Meyer (1990, 1993), Meyer e Allen (1991), Meyer, Allen e Smith (1993).

Esta estrutura tri-dimensional recebe suporte empírico em várias fontes:

. Os modelos tri-fatoriais ajustam-se satisfatoriamente aos dados (Allen e Meyer, 1990, 1996; Meyer, Allen e Smith, 1993; Hackett, Bycio e Hausdorf, 1994; Pavuls, 2000), sendo esta evidência igualmente detectada em amostras portuguesas e brasileiras (por exemplo, Medeiros, 1997; Carochinho, 1998; Rocha e Bastos, 2000; Siqueira, 2001).

. Os antecedentes de cada faceta são distintos (Allen e Meyer, 1990, 1996, 2000; Meyer e Allen, 1991; Hackett, Bycio e Hausdorf, 1994; Meyer, 1997; 
Medeiros e Enders, 1998; Finegan, 2000). Por exemplo, as percepções positivas de justiça explicam o comprometimento afetivo (e, em menor grau, o normativo) mas não o instrumental. Algumas variáveis como o feedback do desempenho na função, a dificuldade dos objetivos e o desafio inerente ao cargo explicam o comprometimento normativo e, especialmente o afetivo, mas relacionam-se negativamente com o instrumental.

As conseqüências de cada faceta são igualmente distintas (Allen e Meyer, 1996, 2000; Meyer, 1997; Meyer e Herscovitch, 2001). Por exemplo, o comprometimento afetivo (e, menos intensamente, o normativo) induz menores intenções de abandonar a organização, menos abandonos efetivos, mais baixos índices de absentismo, mais comportamentos de cidadania e superior desempenho - mas isso não é claro no que concerne ao instrumental. Em suma: a vontade dos indivíduos de contribuírem para os objetivos organizacionais parece ser influenciada pela natureza do laço psicológico que os liga à organização. É mais provável que exerçam elevados esforços de desempenho quando desejam pertencer à organização, do que quando se sentem obrigados ou necessidade de nela se inserirem.

\section{As Percepções de Justiça como um dos Antecedentes do Comprometimento}

A noção (teoricamente aduzida e empiricamente demonstrada) de que o comprometimento organizacional é relevante para o desempenho individual e organizacional tem conduzido numerosos investigadores a estudar os seus potenciais antecedentes. Os dados disponíveis são ricamente prolixos. Numa tentativa de síntese, baseada em múltiplas contribuições (Meyer e Allen, 1991; McFarlin e Sweeney, 1992; Moorman, Niehoff e Organ, 1993; Sweeney e McFarlin, 1993, 1997; Allen e Meyer, 1996; Podsakoff, Mackenzie e Bommer, 1996; Bolon, 1997; Meyer, 1997; Schaubroeck, May e Brown, 1997; MacKenzie, Podsakoff e Ahearne, 1998; Medeiros e Enders, 1998; Mossholder, Bennett e Martin, 1998; Caetano e Vala, 1999; Iverson e Buttigieg, 1999; Maignan, Ferrell e Hult, 1999; Finegan, 2000; Grunberg, Anderson-Connolly e Greenberg, 2000), pode afirmarse o seguinte:

. Entre os antecedentes que mais explicam (positivamente) o comprometimento normativo e, especialmente, o afetivo encontram-se a afetividade positiva, a liderança transformacional, o apoio organizacional percebido, a receptividade da gestão às sugestões dos empregados, o apoio do supervisor e dos colegas, a clareza do papel, a recepção de feedback relativo ao desempenho, as funções 
enriquecidas/desafiantes, a percepção de que os valores organizacionais têm orientação humanizada e visionária, a percepção de que as organizações atuam de modo socialmente responsável (econômica, legal, ética e filantropicamente) e as percepções de justiça.

- A maior parte destes antecedentes correlacionam-se nula ou negativamente com o comprometimento instrumental.

O estudo empírico que alicerça o presente trabalho debruça-se sobre um desses antecedentes: as percepções de justiça. Sucede que este construto também é multidimensional, sendo comum a divisão em três facetas. A justiça distributiva (Adams, 1965) foi a que primeiramente suscitou a atenção dos cientistas organizacionais. Focaliza-se no conteúdo, isto é, na justiça dos fins alcançados ou obtidos (por exemplo, salários, classificações obtidas nas avaliações de desempenho, sanções disciplinares). A justiça procedimental (Thibaut e Walker, 1975; Lind e Tyler, 1988) focaliza-se no processo, ou seja, na justiça dos meios usados para alcançar tais fins. A justiça interacional (por exemplo, Bies e Moag, 1986; Tyler e Bies, 1990; Greenberg, 1993; Rego, 2000b, 2002) reflete a qualidade da interação com os decisores (O gestor age com dignidade e respeito? Oferece justificativas aos indivíduos afetados pelas decisões?).

Alguns dados teóricos e empíricos (Greenberg e McCarty, 1990; Greenberg, 1993; Cropanzano e Greenberg, 1997; Greenberg e Lind, 2000; Konovsky, 2000; Rego, 2000a, 2000b, 2002) sugerem ainda que se distingam, no âmbito da justiça interacional, as facetas social/interpessoal e informacional. A primeira representa o grau em que o superior adota tratamento digno e respeitador. A evidência empírica (Greenberg, 1993) sugere que, quando esse tratamento ocorre, as percepções de justiça são incrementadas, o grau de aceitação das decisões aumenta, e várias reações positivas emergem. A justiça informacional reflete o fornecimento de informações e a explicação/justificativa das decisões. É relevante porque as pessoas esperam que os seus superiores lhes prestem explicações (adequadas, lógicas, sinceras) para as decisões que tomam, particularmente quando os resultados são desfavoráveis (Greenberg, 1993; Cropanzano e Greenberg, 1997). Os estudos sugerem que as explicações/ justificativas mitigam as reações negativas dos indivíduos às percepções de injustiça ou resultados desfavoráveis.

Uma das razões mais vigorosas para autonomizar as várias facetas encontrase no fato delas explicarem diferentemente as reações atidudinais e comportamentais dos membros organizacionais - incluindo o comprometimento. A hipótese subjacente ao presente trabalho é a de que, em relação à justiça distributiva, as facetas procedimental e interacional predominarão na explicação do comprometimento (especialmente o afetivo e o normativo). A premissa 
baseia-se na tese dos efeitos diferenciais (Lind e Tyler, 1988; Greenberg, 1990; McFarlin e Sweeney, 1992; Sweeney e McFarlin, 1993), vastamente enunciada nos estudos organizacionais. Nos seus termos, a justiça procedimental/ interacional e a distributiva têm diferentes consequências: a procedimental relaciona-se com as avaliações do sistema organizacional (refletidas em variáveis como a lealdade à organização, o comprometimento organizacional e a confiança), enquanto a distributiva se associa preferencialmente com a satisfação com os resultados específicos em questão ou os resultados de determinada decisão. Esta noção recebeu apoio empírico em vários estudos (Folger e Konovsky, 1989; Kim e Mauborgne, 1991, 1996; McFarlin e Sweeney, 1992; Sweeney e McFarlin, 1993).

Um dos reflexos mais emblemáticos desta tese está patenteado num modelo de Kim e Mauborgne (1997), visando explicar como as diferentes facetas da justiça conduzem a diferentes atitudes e comportamentos individuais e, assim, a distintos níveis de desempenho organizacional. Sugerem os autores que as pessoas, quando percebem que são distributivamente justiçadas, experimentam sentimentos de satisfação, o que as induz a executar aquilo que delas é esperado. Todavia, quando denotam percepções de justiça procedimental/interacional elevadas, experimentam laços de comprometimento afetivo e sentimentos de confiança para com a organização e os seus decisores. Assim se sentem reconhecidas pelo seu valor pessoal, emocional e intelectual. Conseqüentemente, disponibilizam-se para ir mais além do que é esperado, e para adotar comportamentos de cidadania (Rego, 2002). Estas duas vias conduzem a níveis de desempenho organizacional distintos, sendo mais frutuosos os que têm a gênese na via procedimental/interacional.

Este artigo não almeja expandir-se para a explicação do desempenho organizacional, mas pretende pesquisar se, efetivamente, o comprometimento é mais explicado pela justiça procedimental e interacional do que pela distributiva. Adicionalmente, visa comparar o poder explicativo das duas facetas interacionais (interpessoal/social e informacional). Esta é uma matéria de grande relevância, pois são parcos os estudos que consagram estas duas facetas da justiça (Konovsky, 2000; Rego, 2002).

\section{Metodologia}

O estudo foi levado a cabo em duas fases. A primeira foi realizada no Brasil, tendo sido inquirida uma amostra de conveniência constituída por 229 indivíduos (60\% do sexo masculino): $78 \%$ oriundos de nove empresas privadas e $22 \%$ de 
quatro instituições públicas, todas operando no Município do Rio de Janeiro. A idade média é de 32.3 anos, e a antiguidade média está em 5.53 anos, não se detectando diferenças significativas entre as médias referentes a cada sexo.

Os inquiridos foram convidados a assinalar as suas percepções de justiça num questionário contendo 17 itens/indicadores que Rego (2000a, 2000c), após pesquisa na literatura e adaptação, havia usado. A escolha desses indicadores foi realizada do seguinte modo: (1) selecionaram-se os cinco indicadores distributivos e os quatro procedimentais que haviam remanescido na análise fatorial confirmatória (AFC) executada por Rego; (2) dos cinco itens interacionais emergentes dessa AFC, selecionaram-se quatro, tendo sido eliminado o que denotava menor carga fatorial (desses quatro, três eram de natureza interpessoal e um informacional); (3) foram depois colhidos mais quatro indicadores atinentes à justiça interacional (três informacionais e um interpessoal), que constavam da análise fatorial das componentes principais também realizada por Rego. Aos inquiridos foi apresentada uma escala tipo Likert de 6 pontos (1: completamente falso a 6: completamente verdadeiro).

Os dados foram então submetidos a uma AFC (método da máxima verosimilhança), de acordo com o modelo de quatro fatores antes exposto: distributivo, procedimental, interpessoal, informacional. Para melhorar os índices de ajustamento, removeram-se alguns indicadores, de acordo com os índices de modificação e os resíduos estandartizados (Byrne, 1998). O modelo remanescente integra os 14 itens que a Tabela 1 consagra (do qual constam também os dados psicométricos respeitantes à amostra portuguesa, inquirida na segunda fase do estudo). Aí se pode verificar que os respectivos índices de ajustamento são bastante satisfatórios (Joreskog e Sorbom, 1993; Byrne, 1998). Foram depois testados três modelos adicionais. O primeiro englobava três fatores, resultando da agregação dos dois fatores interacionais (interpessoal e informacional). $\mathrm{O}$ segundo englobava dois fatores, resultando da agregação numa só dimensão do fator procedimental e dos dois fatores interacionais. O terceiro era um modelo uni-dimensional, com todos os indicadores agrupados. Globalmente, o tetra-modelo ajusta-se mais satisfatoriamente aos dados do que o modelo tri-dimensional, embora as diferenças entre os índices sejam reduzidas. Os modelos de dois e um fator denotam índices de ajustamento insatisfatórios (por exemplo, o GFI cifrou-se em 0.81 e 0.64, respectivamente). Foram então selecionados para análise os modelos de três e quatro dimensões de justiça. Note-se que os Alphas de Cronbach se situam invariavelmente acima de 0.70 (Nunnally, 1978). Os Lambdas ultrapassam sempre o patamar de 0.50, situandose quase invariavelmente acima de 0.70 . 
Tabela 1: Análise Fatorial Confirmatória Aplicada aos Dados de Justiça

\begin{tabular}{|c|c|c|c|c|}
\hline & \multicolumn{2}{|c|}{$\begin{array}{c}\mathbf{1}^{\text {a }} \text { fase do } \\
\text { estudo } \\
\text { (amostra } \\
\text { brasileira) }\end{array}$} & \multicolumn{2}{|c|}{$\begin{array}{c}2^{\mathbf{a}} \text { fase do } \\
\text { estudo } \\
\text { (amostra } \\
\text { portuguesa) }\end{array}$} \\
\hline & $\begin{array}{c}\mathbf{3} \\
\text { fatores }\end{array}$ & $\begin{array}{c}4 \\
\text { fatores }\end{array}$ & $\begin{array}{c}3 \\
\text { fatores }\end{array}$ & $\begin{array}{c}4 \\
\text { fatores }\end{array}$ \\
\hline Justiça distributiva & $(0.86)$ & $(0.86)$ & $(0.89)$ & $(0.89)$ \\
\hline Em geral, as recompensas que recebo são justas. & 0.66 & 0.66 & 0.71 & 0.71 \\
\hline $\begin{array}{l}\text { Se considerar os restantes salários pagos nesta organização, reconheço que o meu salário é } \\
\text { justo. }\end{array}$ & 0.78 & 0.78 & 0.78 & 0.78 \\
\hline Se tiver em conta a minha experiência, sinto-me justamente recompensado. & 0.77 & 0.77 & 0.91 & 0.91 \\
\hline Tendo em conta o meu esforço, julgo que sou recompensado justamente. & 0.91 & 0.91 & 0.91 & 0.91 \\
\hline Justiça procedimental & $(0.74)$ & (0.74) & $(0.83)$ & $(0.83)$ \\
\hline A minha organização tem um mecanismo que permite aos empregados apelarem das decisões. & 0.57 & 0.57 & 0.79 & 0.79 \\
\hline $\begin{array}{l}\text { As questões que os empregados colocam a respeito da remuneração e da avaliação de } \\
\text { desempenho são normalmente respondidas pronta e satisfatoriamente. }\end{array}$ & 0.78 & 0.78 & 0.75 & 0.75 \\
\hline $\begin{array}{l}\text { Por meio de vários canais, a minha organização tenta compreender as opiniões dos } \\
\text { empregados relativamente às decisões e políticas de remuneração. }\end{array}$ & 0.78 & 0.79 & 0.86 & 0.86 \\
\hline Justiça interacional & $(0.93)$ & & $(0.95)$ & \\
\hline Justiça interpessoal/social & & $(0.89)$ & & $(0.88)$ \\
\hline O meu superior mostra interesse genuíno em ser justo comigo. & 0.84 & 0.86 & 0.88 & 0.90 \\
\hline O meu superior é completamente sincero e franco comigo. & 0.85 & 0.88 & 0.89 & 0.90 \\
\hline O meu superior trata-me com respeito e consideração. & 0.79 & 0.82 & 0.74 & 0.74 \\
\hline Justiça informacional & & $(0.89)$ & & $(0.92)$ \\
\hline $\begin{array}{l}\text { O meu superior fornece-me informações acerca do modo como eu estou a desempenhar as } \\
\text { minhas funções, permitindo-me aprender a fazer melhor o meu trabalho. }\end{array}$ & 0.82 & 0.81 & 0.83 & 0.83 \\
\hline Ao decidir sobre o meu trabalho, o meu superior dá-me explicações com sentido para mim. & 0.77 & 0.78 & 0.90 & 0.90 \\
\hline O meu superior oferece justificação adequada para as decisões relativas ao meu trabalho. & 0.83 & 0.84 & 0.86 & 0.87 \\
\hline $\begin{array}{l}\text { O meu superior explica muito claramente qualquer decisão relacionada com as minhas } \\
\text { funções. }\end{array}$ & 0.85 & 0.87 & 0.87 & 0.87 \\
\hline \multicolumn{5}{|l|}{ Índices de ajustamento } \\
\hline Qui-quadrado/Graus de liberdade & 2.8 & 2.4 & 1.80 & 1.74 \\
\hline Root mean square error of approximation & 0.088 & 0.078 & 0.058 & 0.057 \\
\hline Goodness of fit index & 0.89 & 0.90 & 0.92 & 0.93 \\
\hline Adjusted goodness of fit index & 0.84 & 0.86 & 0.89 & 0.89 \\
\hline Comparative fit index & 0.94 & 0.95 & 0.98 & 0.98 \\
\hline Incremental fit index & 0.94 & 0.95 & 0.98 & 0.98 \\
\hline Relative fit index & 0.89 & 0.90 & 0.94 & 0.94 \\
\hline
\end{tabular}

Nota: os valores representam as cargas factoriais, exceptuando os colocados entre parêntesis, que representam os Alphas de Cronbach.

Para medir o comprometimento organizacional, os inquiridos foram convidados a responder a um questionário contendo 28 indicadores usados previamente por Rego (2003), após pesquisa na literatura (apresentada na seção Bibliografia) e adaptação. Foi-lhes apresentada uma escala tipo Likert de 7 pontos (1: a afirmação não se aplica rigorosamente nada a mim a 7: aplica-se completamente a mim). Os dados foram submetidos a uma análise fatorial das componentes principais, com rotação varimax. Para que a estrutura fatorial fosse clara, foram removidos os indicadores cujas cargas fatoriais eram superiores a 0.50 em mais do que um fator. Usando como critério as componentes com valores próprios superiores a 1, emergiu uma estrutura de três fatores (correspondentes às tradicionais dimensões afetiva, normativa e instrumental), contendo 16 indicadores e explicando $58 \%$ da variância total (vide Tabela 2). Os coeficientes de consistência interna são invariavelmente superiores a 0.80 . 


\section{Tabela 2: Análise Fatorial das Componentes Principais dos Dados de Comprometimento Referentes à Amostra Brasileira*}

\begin{tabular}{|c|c|c|c|}
\hline & $\begin{array}{l}\text { Fator } 1 \\
\text { Afetivo }\end{array}$ & $\begin{array}{c}\text { Fator } 2 \\
\text { Normativo }\end{array}$ & $\begin{array}{c}\text { Fator } 3 \\
\text { Instrumental }\end{array}$ \\
\hline Lamento ter vindo trabalhar para esta organização. & -0.56 & -0.12 & 0.13 \\
\hline Tenho forte ligação de simpatia por esta organização. & 0.85 & 0.19 & -0.10 \\
\hline Importo-me realmente com o destino da minha organização. & 0.74 & -0.04 & -0.04 \\
\hline Sinto que existe forte ligação afetiva entre mim e a minha organização. & 0.66 & 0.39 & 0.06 \\
\hline Tenho orgulho em dizer a outras pessoas que faco parte desta organização. & 0.69 & 0.20 & -0.28 \\
\hline $\begin{array}{l}\text { Não deixaria a minha organização agora porque sinto obrigações para com as pessoas } \\
\text { que aqui trabalham. }\end{array}$ & 0.31 & 0.69 & -0.13 \\
\hline Sinto-me em dívida para com a minha organização. & 0.00 & 0.69 & 0.23 \\
\hline $\begin{array}{l}\text { Sinto que, se recebesse uma oferta de melhor emprego, não seria correto deixar a } \\
\text { minha organização. }\end{array}$ & 0.18 & 0.81 & -0.01 \\
\hline Sinto que tenho poucas alternativas de emprego se deixar esta organização. & -0.17 & 0.07 & 0.72 \\
\hline $\begin{array}{l}\text { Mantenho-me nesta organização porque sinto que tenho poucas oportunidades noutras } \\
\text { organizações. }\end{array}$ & -0.12 & -0.09 & 0.72 \\
\hline Não abandono esta organização devido às perdas que me prejudicariam. & 0.05 & 0.07 & 0.76 \\
\hline Percentagem de variância explicada & $29.1 \%$ & $19.1 \%$ & $10 \%$ \\
\hline Valores próprios & 4.65 & 3.06 & 1.60 \\
\hline Alpha de Cronbach & 0.83 & 0.81 & 0.81 \\
\hline
\end{tabular}

Nota: exceptuando os que estão escritos a itálico, os valores representam as cargas factoriais; * KMO: 0.84; teste de esfericidade de Bartlett: 1403.87 ( $\mathrm{p}=0.000)$.

Registre-se que esta estrutura tri-dimensional não corrobora o esquema factorial que Rego (2003) obtivera. O investigador testara modelos de três, quatro, cinco e seis factores, tendo chegado a índices de ajustamento mais satisfatórios para os três últimos modelos. A evidência aqui exposta sugere, distintamente, que o modelo tradicional de Meyer e Allen continua a receber guarida empírica, resistindo às contingências amostrais e culturais.

A segunda fase do estudo foi concretizada com uma amostra de conveniência abarcando 236 indivíduos, oriundos de duas organizações privadas e de três instituições públicas operando em Portugal. 46\% são do sexo masculino. A idade média é de 34.4 anos, e a antiguidade média está em 9.7 anos, não se tendo detectado diferenças significativas entre as médias referentes a cada sexo.

Para medir as percepções de justiça, os indivíduos foram convidados a responder ao mesmo questionário que o aplicado à amostra brasileira. Os dados foram submetidos a AFC (método da máxima verosimilhança), tendo sido usados os mesmos procedimentos anteriormente expostos para a amostra do Brasil. Foram selecionados para posterior análise os modelos de três e quatro dimensões, por denotarem índices de ajustamento satisfatórios (vide Tabela 1). Registre-se que, reiteradamente, os Alphas de Cronbach se situam acima de 0.70. Os Lambdas 
ultrapassam sempre o patamar de 0.50, situando-se quase invariavelmente acima de 0.70 .

A medição do comprometimento organizacional foi levada a cabo com um questionário contendo os 16 indicadores que haviam remanescido da análise fatorial exploratória aplicada à amostra portuguesa. Os dados foram submetidos a uma AFC (método da máxima verosimilhança), tendo sido testado o modelo de três fatores que essa análise anterior sugerira. Visando incrementar os índices de ajustamento, foram removidos dois indicadores (Byrne, 1998). Tal como revela a Tabela 3, o modelo ajusta-se de modo bastante satisfatório aos dados - o que aliás é igualmente notório para a amostra brasileira. Também aqui, os Lambdas são sempre superiores a 0.50, e os Alphas de Cronbach ultrapassam o patamar recomendável de 0.70 .

\section{Tabela 3: Análises Fatoriais Confirmatórias Aplicadas aos Dados de Comprometimento}

\begin{tabular}{|c|c|c|}
\hline & $\begin{array}{c}\text { Amostra } \\
\text { portuguesa }\end{array}$ & $\begin{array}{r}\text { Amostra } \\
\text { brasileira }\end{array}$ \\
\hline Tenho forte ligação de simpatia por esta organização. & 0.89 & 0.88 \\
\hline Importo-me realmente com o destino da minha organização. & 0.55 & 0.59 \\
\hline Sinto que existe forte ligação afetiva entre mim e a minha organização. & 0.78 & 0.69 \\
\hline Tenho orgulho em dizer a outras pessoas que faço parte desta organização. & 0.71 & 0.69 \\
\hline Mesmo que isso me trouxesse vantagens, sinto que não deveria abandonar a minha organização agora. & 0.81 & 0.74 \\
\hline Não deixaria a minha organização agora porque sinto obrigações para com as pessoas que aqui trabalham. & 0.76 & 0.72 \\
\hline Sinto-me em dívida para com a minha organização. & 0.65 & 0.51 \\
\hline Sinto que, se recebesse uma oferta de melhor emprego, não seria correto deixar a minha organização. & 0.79 & 0.74 \\
\hline Comprometimento instrumental & $(0.80)$ & $(0.81)$ \\
\hline Mantenho-me nesta organização porque sinto que não conseguiria facilmente entrar noutra organização. & 0.62 & 0.69 \\
\hline \multicolumn{3}{|l|}{ Índices de ajustamento } \\
\hline Qui-quadrado/Graus de liberdade & 2.1 & 2.3 \\
\hline Root mean square error of approximation & 0.068 & 0.075 \\
\hline Goodness of fit index & 0.91 & 0.90 \\
\hline Adjusted goodness of fit index & 0.88 & 0.86 \\
\hline Comparative fit index & 0.95 & 0.92 \\
\hline Incremental fit index & 0.95 & 0.92 \\
\hline Relative fit index & 0.88 & 0.83 \\
\hline
\end{tabular}

Nota: os valores situados à frente de cada item representam as cargas factoriais, exceptuando os colocados entre parêntesis, que representam os Alphas de Cronbach.

Para testar a relação entre as percepções de justiça e o comprometimento, foram calculados coeficientes de correlação, executadas análises de regressão linear simples e efectuada uma análise de clusters. Para que os dados referentes às duas amostras fossem comparáveis, todos os indivíduos foram cotados por meio dos itens remanescentes das AFC. Isso significa que, no caso concreto do comprometimento, os indivíduos da amostra brasileira foram cotados por meio 
dos 14 indicadores confirmatórios, e não dos 16 que a análise fatorial das componentes principais sugerira.

Atendendo à pequena diferença entre os índices de ajustamento dos modelos de três e quatro dimensões de justiça (vide Tabela 1), efetuaram-se análises de regressão com o recurso a ambos os modelos. O objetivo era o de saber: (1) qual dos dois modelos facultava maiores poderes explicativos do comprometimento; (2) se as duas facetas interacionais explicavam diferentemente as várias dimensões do comprometimento. Os resultados sugerem que a consideração separada das duas vertentes denota um panorama explicativo de maior valia.

\section{Resultados}

A Tabela 4 exibe as médias, desvios-padrões e correlações. Qualquer que seja a amostra considerada, as dimensões de justiça correlacionam-se positivamente entre si, sendo mais fortes as relações entre as duas facetas interacionais. $\mathrm{O}$ comprometimento afetivo e normativo também se relacionam positivamente entre si, mas não com o comprometimento instrumental. Em geral, as percepções de justiça relacionam-se positivamente com o comprometimento afetivo e normativo, mas nula ou negativamente com o instrumental.

\section{Tabela 4: Médias, Desvios-Padrões e Correlações}

\begin{tabular}{|c|c|c|c|c|c|c|c|c|}
\hline & Média & D-padrão & 1 & 2 & 3 & 4 & 5 & 6 \\
\hline 1. J. Distributiva & $\begin{array}{l}3.7 \\
3.0\end{array}$ & $\begin{array}{l}1.3 \\
1.3\end{array}$ & - & & & & & \\
\hline 2. J. Procedimental & $\begin{array}{l}3.4 \\
2.8\end{array}$ & $\begin{array}{l}1.2 \\
1.3\end{array}$ & $\begin{array}{l}0.60^{* * *} \\
0.65^{* * *}\end{array}$ & - & & & & \\
\hline 3. J. Interpessoal & $\begin{array}{l}4.7 \\
4.3\end{array}$ & $\begin{array}{l}1.2 \\
1.2\end{array}$ & $\begin{array}{l}0.35^{* * *} \\
0.42^{* * *}\end{array}$ & $\begin{array}{l}0.49^{* * *} \\
0.61^{* * *}\end{array}$ & - & & & \\
\hline 4. J. Informacional & $\begin{array}{l}4.4 \\
3.9\end{array}$ & $\begin{array}{l}2.0 \\
1.3\end{array}$ & $\begin{array}{l}0.42^{* * *} \\
0.42^{* * *}\end{array}$ & $\begin{array}{l}0.55^{* * *} \\
0.65^{* * *}\end{array}$ & $\begin{array}{l}0.83^{* * *} \\
0.89^{* * *}\end{array}$ & - & & \\
\hline 5. C. Afetivo & $\begin{array}{l}4.9 \\
4.7\end{array}$ & $\begin{array}{l}1.3 \\
1.2\end{array}$ & $\begin{array}{l}0.35^{* * *} \\
0.35^{* * *}\end{array}$ & $\begin{array}{l}0.42^{* * *} \\
0.46^{* * *}\end{array}$ & $\begin{array}{l}0.49^{* * *} \\
0.40^{* * *}\end{array}$ & $\begin{array}{l}0.46^{* * *} \\
0.37 * * *\end{array}$ & - & \\
\hline 6. C. Normativo & $\begin{array}{l}3.1 \\
3.1\end{array}$ & $\begin{array}{l}1.3 \\
1.3\end{array}$ & $\begin{array}{l}0.32^{* * *} \\
0.53^{* * *}\end{array}$ & $\begin{array}{l}0.36^{* * *} \\
0.55^{* * *}\end{array}$ & $\begin{array}{l}0.23^{* * *} \\
0.46^{* * *}\end{array}$ & $\begin{array}{l}0.31^{* * *} \\
0.47^{* * *}\end{array}$ & $\begin{array}{l}0.47^{* * *} \\
0.60^{* * *}\end{array}$ & - \\
\hline 7. C. Instrumental & $\begin{array}{l}2.7 \\
2.8\end{array}$ & $\begin{array}{l}1.1 \\
1.1\end{array}$ & $\begin{array}{l}0.01 \\
-0.11\end{array}$ & $\begin{array}{l}-0.08 \\
-0.27 * * *\end{array}$ & $\begin{array}{l}-0.16^{*} \\
-0.18^{* *}\end{array}$ & $\begin{array}{l}-0.09 \\
-0.20^{* *}\end{array}$ & $\begin{array}{l}-0.16^{*} \\
-0.11\end{array}$ & $\begin{array}{l}0.03 \\
0.06\end{array}$ \\
\hline
\end{tabular}

Nota: a primeira linha refere-se à amostra brasileira, e a segunda à amostra portuguesa; ${ }^{*} \mathrm{p}<0.05$; $* * \mathrm{p}<0.01 ; * * * \mathrm{p}<0.001$.

Na Tabela 5 estão expostos os resultados das análises de regressão linear simples, denotando o poder explicativo das percepções de justiça para o comprometimento. 
Para aferir a pertinência da consideração separada das duas facetas interacionais de justiça versus a sua consideração agregada, foram calculadas regressões para as duas possibilidades. Os resultados sugerem o seguinte: (1) as percepções de justiça explicam o comprometimento afetivo e normativo, mas é reduzido o seu poder explicativo do comprometimento instrumental; (2) a justiça distributiva tende a explicar o comprometimento normativo, mas não as outras duas dimensões; (3) embora com algumas nuances, o comprometimento afetivo e normativo são fundamentalmente explicados pela justiça procedimental e pela interacional; (4) a consideração separada das duas vertentes interacionais permite aumentar as variâncias explicadas do comprometimento; (5) apenas a vertente interpessoal da justiça interacional tende a explicar o comprometimento, mas a informacional denota poder explicativo da faceta normativa na amostra brasileira.

\section{Tabela 5: Regressões - Poder Explicativo das Percepções de Justiça para o Comprometimento}

\begin{tabular}{|c|c|c|c|c|c|c|c|c|c|c|c|c|}
\hline & \multicolumn{6}{|c|}{ Amostra brasileira } & \multicolumn{6}{|c|}{ Amostra portuguesa } \\
\hline & \multicolumn{2}{|c|}{ Afetivo } & \multicolumn{2}{|c|}{ Normativo } & \multicolumn{2}{|c|}{ Instrumental } & \multicolumn{2}{|c|}{ Afetivo } & \multicolumn{2}{|c|}{ Normativo } & \multicolumn{2}{|c|}{ Instrumental } \\
\hline J. Distributiva & 0.10 & 0.11 & $0.14 \#$ & $0.14 \#$ & 0.09 & 0.08 & 0.11 & 0.11 & $0.30^{* * *}$ & $0.30^{* * *}$ & 0.07 & 0.07 \\
\hline J. Procedimental & $0.17^{*}$ & $0.18^{*}$ & $0.22 * *$ & $0.21 *$ & -0.06 & -0.07 & $0.27^{* *}$ & $0.28^{* *}$ & $0.21^{* *}$ & $0.21^{* *}$ & $-0.28 * *$ & $-0.27^{* *}$ \\
\hline J. Interacional & $0.36^{* * *}$ & & 0.11 & & -0.13 & & $0.18^{*}$ & & $0.22 * *$ & & -0.06 & \\
\hline J. Interpessoa & & $0.32^{* *}$ & & -0.13 & & $-0.28^{*}$ & & $0.25^{*}$ & & 0.06 & & 0.06 \\
\hline J. Informaciona & & 0.05 & & $0.25^{*}$ & & 0.15 & & -0.07 & & 0.16 & & -0.12 \\
\hline $\mathrm{F}$ & $29.2 * * *$ & $22.8^{* * *}$ & $13.9^{* * *}$ & $11.3^{* * *}$ & 1.56 & $2.15 \#$ & $23.7^{* * *}$ & $18.4^{* * *}$ & $46.6^{* * *}$ & $34.8^{* * *}$ & $6.2 * * *$ & $4.75^{* * *}$ \\
\hline $\mathrm{R}^{2}$ ajustado & $27 \%$ & $28 \%$ & $15 \%$ & $15 \%$ & $1 \%$ & $2 \%$ & $23 \%$ & $23 \%$ & $37 \%$ & $37 \%$ & & $6 \%$ \\
\hline
\end{tabular}

Nota: $\# \mathrm{p}<0.10 ;{ }^{*} \mathrm{p}<0.05 ;{ }^{* *} \mathrm{p}<0.01 ;{ }^{* * *} \mathrm{p}<0.001$.

A Tabela 6 apresenta os resultados de uma análise de clusters (método Ward; quadrado da distância euclidiana), efetuada sobre as duas amostras, com base nos dados de comprometimento. Foram extraídos sete agrupamentos, posteriormente comparados (Teste Sheffé) de acordo com as cotações em comprometimento e em percepções de justiça. O teste do qui-quadrado sugere que o sexo e a origem da amostra são independentes dos agrupamentos, ou seja, homens e mulheres distribuem-se uniformemente pelos vários grupos, o mesmo ocorrendo com os indivíduos brasileiros e portugueses. Também não foram detectadas diferenças significativas entre os grupos no que concerne à idade e à antiguidade. Os agrupamentos podem ser assim denominados e caracterizados:

- Os indivíduos do grupo 1 foram denominados psicologicamente ausentes por denotarem muito baixas cotações em todas as dimensões do comprometimento. Não se sentem ligados à organização do ponto de vista afetivo, nem normativo, nem instrumental. São também os que expressam percepções de justiça mais negativas. 
. As pessoas do grupo 2 foram designadas instrumentalmente ligadas porque, embora denotem pontuações bastante abaixo da média nas dimensões afetiva e normativa, apresentam, entre todos os grupos, a mais elevada cotação na vertente instrumental. Expressam percepções de justiça muito similares às do grupo anterior, embora ligeiramente menos negativas.

. Os elementos do grupo 3 apresentam laços tênues, isto é, revelam um razoável comprometimento afetivo, mas fracas ligações normativas e instrumentais. Denotam percepções de justiça bastante modestas, embora mais positivas do que as perfilhadas pelos dois grupos anteriores.

- Os indivíduos do grupo 4 foram denominados de afetiva e instrumentalmente ligados porque, embora revelem fraca ligação normativa, denotam elevado comprometimento afetivo e a segunda mais elevada cotação na faceta instrumental. Expressam percepções de justiça muito próximas das que caracterizam o grupo anterior.

- O grupo 4 é composto por pessoas com laços plurais: os laços afetivo e normativo são relativamente fortes, e o comprometimento instrumental é um dos mais elevados entre os vários agrupamentos. Denotam percepções de justiça ligeiramente mais positivas dos que as relativas aos dois grupos anteriores.

. Os indivíduos do grupo 6 foram designados afetivamente comprometidos mas sem sentido do dever de lealdade porque, embora afetivamente ligados à organização e sem ligação instrumental notória, revelam fraco sentido do dever de lealdade. Expressam percepções de justiça semelhantes às do agrupamento anterior.

- Finalmente, as pessoas inseridas no grupo 7 receberam a denominação de afetivamente comprometidas e leais. São as que denotam os mais fortes níveis de comprometimento afetivo e normativo, e revelam laço instrumental muito fraco. São os que manifestam as mais positivas percepções de justiça.

Tendencialmente, os dados revelam o seguinte: (1) os indivíduos caracterizamse pelo modo como neles se combinam os três laços psicológicos; (2) elevadas orientações numa das facetas do comprometimento podem coabitar com fracas orientações noutras facetas, e vice-versa; (3) os indivíduos com fortes laços afetivos e normativos e com baixo comprometimento instrumental são os que tendem a revelar percepções de justiça mais positivas. 
Tabela 6: Agrupamentos dos Indivíduos de acordo com os seu Níveis de Comprometimento e Respectivas Percepções de Justiça

\begin{tabular}{|c|c|c|c|c|c|c|c|c|}
\hline & $\begin{array}{c}\text { Grupo 1 } \\
\text { Psicologi- } \\
\text { camente } \\
\text { ausentes } \\
\\
(\mathrm{n}=81)\end{array}$ & $\begin{array}{c}\text { Grupo 2 } \\
\text { Instrumental- } \\
\text { mente ligados }\end{array}$ & $\begin{array}{c}\text { Grupo } 3 \\
\text { Com laços } \\
\text { tênues }\end{array}$ & $\begin{array}{c}\text { Grupo } 4 \\
\text { Afetiva e } \\
\text { instrumental- } \\
\text { mente ligados } \\
\\
(\mathrm{n}=38)\end{array}$ & $\begin{array}{c}\text { Grupo } 5 \\
\text { Com laços } \\
\text { plurais }\end{array}$ & $\begin{array}{c}\text { Grupo 6 } \\
\text { Afetivamente } \\
\text { comprometidos } \\
\text { mas sem sentido } \\
\text { do dever de } \\
\text { lealdade } \\
(\mathrm{n}=79)\end{array}$ & $\begin{array}{c}\text { Grupo } 7 \\
\text { Afetivamente } \\
\text { comprometidos } \\
\text { e leais } \\
\\
(\mathrm{n}=39)\end{array}$ & $\begin{array}{c}\text { Quantidade } \\
\text { de pares de } \\
\text { grupos } \\
\text { diferentes* }\end{array}$ \\
\hline C. Afetivo & 3.2 & 3.9 & 4.7 & 5.9 & 5.1 & 5.9 & 6.2 & 17 \\
\hline C. Normativo & 1.9 & 2.2 & 2.8 & 3.1 & 4.6 & 3.0 & 5.4 & 17 \\
\hline C. Instrumental & 2.1 & 4.1 & 2.4 & 3.6 & 3.5 & 1.9 & 1.8 & 16 \\
\hline J. Distributiva & 2.6 & 2.7 & 3.6 & 3.5 & 3.9 & 3.5 & 4.2 & 8 \\
\hline J. Procedimental & 2.3 & 2.4 & 3.3 & 3.1 & 3.5 & 3.5 & 4.2 & 10 \\
\hline J. Interpessoal & 3.7 & 4.1 & 4.7 & 4.6 & 4.8 & 4.9 & 5.3 & 7 \\
\hline J. Informacional & 3.3 & 3.7 & 4.3 & 4.3 & 4.5 & 4.4 & 5.1 & 9 \\
\hline
\end{tabular}

Nota: *Teste Scheffé $(\mathrm{p}=0.05)$.

\section{Análise, Discussão e Conclusões}

O primeiro elemento digno de menção é o de que os dois instrumentos de medida aqui expostos denotam boas propriedades psicométricas, seja do ponto de vista do ajustamento dos modelos fatoriais, seja no que concerne às consistências internas, seja no que respeita aos poderes preditivos detectados. Esta evidência adquire importância acrescida pelo fato de ser verificável com amostras colhidas em duas culturas distintas.

Segundo aspecto merecedor de atenção: o modelo tri-dimensional do comprometimento (o mais comum na literatura) recebe aqui comprovação empírica. Em terceiro lugar, cabe assinalar que o modelo tetra-dimensional das percepções de justiça revela propriedades psicométricas que recomendam que ele seja seriamente considerado em pesquisas posteriores. Embora não se distinga vincadamente do modelo tri-fatorial (as diferenças entre os índices de ajustamento dos dois modelos são reduzidas), há vantagens em considerá-lo quando se procura explicar o comprometimento organizacional: (1) os poderes explicativos sofrem algum incremento; (2) a faceta interpessoal predomina na explicação do comprometimento afetivo, ao passo que a justiça informacional prepondera na explicação do laço normativo. Em suma, os dados são reveladores de que se justifica plenamente a consideração separada da justiça procedimental e da interacional, mas também a partição da justiça interacional nas vertentes interpessoal e informacional.

Em quarto lugar, importa notar que, embora o panorama empírico relativo seja 
similar para as amostras portuguesa e brasileira, é possível identificar algumas dissemelhanças. Por exemplo, a justiça informacional explica o comprometimento normativo na amostra brasileira, mas não na portuguesa. De modo distinto, o poder explicativo da justiça distributiva para essa faceta do comprometimento é maior na amostra portuguesa do que na brasileira. Uma potencial via explicativa para esta dissonância empírica radica no contexto cultural. Na verdade, existem indicações de que os poderes relativos das várias facetas da justiça não são independentes desse contexto (por exemplo, Bond, Leung e Schwartz, 1992; James, 1993; Farh, Earley e Lin, 1997; Gilliland e Chan, 2001; McFarlin e Sweeney, 2001). Por conseguinte, importa que futuros estudos explorem o papel das contingências culturais, designadamente com o recurso ao tradicional modelo de Hofstede $(1980,1991)$.

Quinta menção meritória: tal como sugere o modelo dos efeitos diferenciais (Lind e Tyler, 1988; Sweeney e McFarlin, 1993), a justiça procedimental/ interacional predomina, relativamente à distributiva, na explicação do comprometimento. Todavia, isso não é tão claro no que concerne ao laço normativo. Parece, pois, que o sentido do dever de lealdade dos indivíduos à organização é fomentado pela justiça distributiva, além de o ser pelas outras facetas. Estes dados, embora reforcem a idéia da enorme relevância da justiça procedimental e interacional para o comprometimento organizacional, apontam para a necessidade de não ser descurada a justiça distributiva. Por outro lado, importa considerar a possibilidade de as várias facetas da justiça interagirem na produção de efeitos atitudinais e comportamentais, cada uma atenuando ou reforçando os efeitos das outras. Embora esse propósito não conste dos objectivos desse artigo, ele merece ser considerado em futuras pesquisas - tal como, aliás, diversa evidência teórica e empírica tem sugerido (Cropanzano e Folger, 1991; Brockner e Wiesenfeld, 1996; Skarlicki e Folger, 1997).

Em jeito de síntese, poder-se-ia então afirmar que brota do estudo uma simples mas lapidar linha orientadora: se as organizações desejam colaboradores mais afetiva e normativamente comprometidos, e menos associados por laços instrumentais, então necessitam de atuar sobre as várias facetas da justiça - isto é, sobre as rosas (procedimental/interacional) mas também sobre o pão (distributiva) (Martin e Harder, 1994; Rego, 2000a, 2000b, 2002). Essa é, porventura, uma das vias mais frutuosas para o fomento do desempenho organizacional, tal como vários autores têm sugerido (por exemplo, Greenberg, 1990; Kim e Mauborgne, 1997; Greenberg e Lind, 2000; Rego, 2000a, 2000b, 2002).

$\mathrm{O}$ sexto aspecto que cabe salientar diz respeito à necessidade de interpretar $\mathrm{o}$ comprometimento dos indivíduos em função do modo peculiar como neles se 
combinam os diferentes laços psicológicos. É certo que as correlações entre as vertentes afetiva e normativa do comprometimento são significativamente elevadas. Todavia, e exemplificando com o sugerido pela análise de clusters (vide Tabela 5), alguns indivíduos combinam elevado pendor afetivo com fraco laço normativo. Globalmente, o que os dados sugerem é que há vantagens em caracterizar as pessoas à luz das suas configurações ou perfis de comprometimento. Ademais, a caracterização destas configurações sublinhadas pela análise de clusters reforça a já referida importância das várias facetas da justiça: as pessoas mais comprometidas afetiva e normativamente com as suas organizações, e menos ligadas instrumentalmente, são as que expressam as mais positivas percepções de justiça - seja ela distributiva, procedimental, interpessoal ou informacional.

Importa notar que a pesquisa comporta algumas limitações. Embora elas não ofusquem a relevância da evidência empírica facultada, recomendam precauções em estudos posteriores:

- As variáveis dependentes e independentes foram colhidas na mesma fonte e no mesmo momento. Esta opção metodológica atrai riscos de variância do método comum (Podsakoff e Organ, 1986), de tal modo que as relações podem estar inflacionadas por um raciocínio do tipo: "sinto-me justamente tratado na minha organização - logo, devo sentir-me comprometido com ela”. Estudos vindouros deverão contornar estes riscos, designadamente mediante obtenção dos dados de justiça e de comprometimento em momentos temporalmente distanciados.

. Não foram consideradas variáveis moderadoras. Estudos posteriores poderão testar se, por exemplo, a antiguidade, o sexo e os níveis de escolaridade moderam as relações entre a justiça e o comprometimento. Será que as pessoas de diferentes antiguidades, de cada sexo, e com diferentes níveis escolares respondem diferentemente às percepções de justiça? Será que o mesmo pode ocorrer com pessoas possuidoras de diferentes características de personalidade?

. Estão ausentes do estudo outras variáveis que poderiam ajudar a compreender as razões pelas quais a justiça explica o comprometimento. A título ilustrativo (ver síntese em Rego [2000a, 2000b, 2002]), mencionam-se a confiança, os contratos relacionais, a relação vinculativa, as trocas sociais e o reconhecimento intelectual e emocional.

Em qualquer caso, o estudo faculta preciosas contribuições em vários domínios. Primeiro, ajuda a compreender mais profundamente a multidimensionalidade das percepções de justiça e do comprometimento organizacional. Segundo, mostra como as percepções de justiça, nas suas diversas facetas, relevam para efeitos 
do modo como as pessoas se ligam psicologicamente às suas organizações. Terceiro, sugere que as tendências empíricas são detectáveis em duas culturas que, embora partilhem laços comuns, são distintas em vários aspectos.

\section{Agradecimento}

Estamos muito gratos aos pareceristas anônimos pelo seus preciosos comentários e sugestões que permitiram enriquecer este artigo.

\section{Bibliografia}

ADAMS, J. S.

Inequity in social exchange. In: BERKOWITZ, L. (Ed.). Advances in experimental social psychology. New York: Academic Press, 1965. v. 2. p. 267-299.

\section{ALLEN, N. J.;}

MEYER, J. P.

The measurement and antecedents of affective, continuance, and normative commitments to the organization.

Journal of Occupational Psychology, v.1, n. 63, p. 1-8, 1990.

Organizational commitment: evidence of career stage effects? Journal of Business Research, n. 26, p. 49-61, 1993.

Affective, continuance, and normative commitment to the organization: an examination of construct validity. Journal of Vocational Behavior, n. 49, p. 252-276, 1996.

Construct validation in organizational behavior research: the case of organizational commitment. In: GOFFIN, R. D.; HELMES, E. (Eds.). Problems and solutions in human assessment: honouring Douglas N. Jackson at seventy. Norwell, MA: Kluwer Academic Publishers, 2000. p. 285-314.

BECKER, T. E.

Foci and bases of commitment: are they distinctions worth making? Academy of Management Journal, v.1, n. 35, p. 232-244, 1992. 
BIES, R. J.;

MOAG, J. S.

Interactional

justice:

communication criteria of fairness.

In: LEWICKI, R. J.; SHEPPARD,

B. H.; BAZERMAN, M. H.

(Eds.). Research on negotiation in organizations. New York: JAI Press, 1986. v. 1. p. 43-55.

BISHOP, J.;

SCOTT, K.;

BURROUGHS, S.

Support, commitment, and employee outcomes in a team environment. Journal of Management, v. 6, n. 26, p. 11131132, 2000.

BOLON, D. S.

Organizational citizenship behavior among hospital employees: a multidimensional analysis involving job satisfaction and organizational commitment. Hospital \& Health Services Administration, v. 2, n. 42, p. 221-241, 1997.

BOND, M. H.;

LEUNG, K.;

SCHWARTZ, S.

Explaining choices in procedural and distributive justice across cultures. International Journal of Psychology, v. 27, n. 2, p. 211225, 1992.

BROCKNER, J.;

WIESENFELD, B. M.

An integrative framework for explaining reactions to decisions: interactive effects of outcomes and procedures. Psychological Bulletin, v. 2, n. 120, p. 189-208, 1996.

BURR, R.;

GIRARDI, A.

The interaction between competence and commitment as a predictor of human capital within the firm. Trabalho apresentado na Academy of Management Conference, Washington, D.C., 2001.

BURROUGHS, S. M.;

EBY, L. T.

Psychological sense of community at work: a measurement system and explanatory framework. Journal of Community Psychology, v. 6, n. 26, p. 509532, 1998.

BYRNE, B. M.

Structural equation modeling with lisrel, prelis, and simplis. London: Lawrence Erlbaum, 1998.

CAETANO, A.;

VALA, J.

Efeitos da justiça organizacional percebida sobre a satisfação no trabalho e as opções comportamentais. Psicologia, v.1-2, n. XIII, p. 75-84, 1999. 
CAROCHINHO, J. A.;

NEVES, J. G.;

JESUÍNO, J. C.

Organizational commitment: o conceito e sua medida - adaptação e validação do Organizational Commitment Questionnaire(OCQ) para a cultura organizacional portuguesa. Psicologia: Teoria, Investigação e Prática, n. 3, p. 269-284, 1998.

\section{CLUGSTON, $\mathrm{M}$.}

The mediating effects of multidimensional commitment on job satisfaction and intent to leave. Journal of Organizational Behavior, n. 21, p. 477-486, 2000.

\section{COHEN, A.}

The relationship between commitment forms and work outcomes: a comparison of three models. Human Relations, v. 3, n. 53, p. 387-417, 2000.

CROPANZANO, R.;

GREENBERG, J.

Progress in organizational justice: tunnelling through the maze. In: COOPER, C. L.; ROBERTSON, I. T. (Eds.). International review of industrial and organizational psychology. New York: John Wiley \& Sons, 1997. v. 12. p. 317-372.

DUTTON, J. E.;

DUBERICH, J. M.;

HARQUAIL, C. V.

Organizational images and member identification.
Administrative Science

Quarterly, n. 39, p. 239-263, 1994.

FARH, J.;

EARLEY, P. C.;

LIN, S.

Impetus for action: a cultural analysis of justice and organizational citizenship behavior in Chinese society. Administrative Science Quarterly, v. 42, p. 421-444, 1997.

FINEGAN, J. E.

The impact of person and organizational values on organizational commitment. Journal of Occupational and Organizational Psychology, n. 73, p. 149-169, 2000.

FOLGER, R.;

KONOVSKY, M. A.

Effects of procedural and distributive justice on reactions to pay raise decisions. Academy of Management Review, v.1, n. 32, p. 115-130, 1989.

GILLILAND, S. W.; CHAN, D.

Justice in organizations: theory, methods, and applications. In: ANDERSON, N. et al. (Eds.). Handbook of industrial, work and organizational psychology. London: Sage Publications, 2001. v. 2. p. 143-165. 
GOLEMAN, D.

Trabalhar com inteligência emocional. Lisboa: Temas e Debates, 1999.

GREENBERG, J.

Organizational justice: yesterday, today and tomorrow. Journal of Management, n. 16, p. 399-432, 1990.

The social side of fairness: interpersonal and informational classes of organizational justice. In: CROPANZANO, R. (Ed.). Justice in the workplace: approaching fairness in human resource management. Hillsdale, NJ: Erlbaum, 1993. p. 79-103.

GREENBERG, J.;

LIND, E. A.

The pursuit of organizational justice: from conceptualization to implication to application. In: COOPER, C. L.; LOCKE, E. A. (Eds.). Industrial and organizational psychology: linking theory with practice. Malden, MA: Blackwell, 2000. p. 72-108.

GREENBERG, J.;

MCCARTY, C. L.

The interpersonal aspects of procedural justice: a new perspective on pay fairness. Labor Law Journal, n. 41, p. 580-586, 1990.
GRUNBERG, L.;

ANDERSON-CONNOLLY, R.;

GREENBERG, E. S.

Surviving layoffs: the effects on organizational commitment and job performance. Work and Occupations, v. 1, n. 27, p. 7-31, 2000.

HACKETT, R. D.;

BYCIO, P.;

HAUSDORF, P. A.

Further assessments of Meyer and Allen's (1991) threecomponent model of organizational commitment. Journal of Applied Psychology, n. 79, p. 15-23, 1994.

HOFSTEDE, G.

Culture's consequences. London: Sage Publications, 1980.

Cultures and organizations: software of the mind. New York: McGraw-Hill, 1991.

IVERSON, R. D.;

BUTTIGIEG, D. M.

Affective, normative and continuance commitment: can the 'right kind' of commitment be managed? Journal of Management Studies, v. 3, n. 36, p. 307-333, 1999. 
JAMES, K.

The social context of organizational justice: cultural, intergroup, and structural effects on justice behaviors and perceptions. In: CROPANZANO, R. (Ed.). Justice in the workplace. London: Lawrence Erlbaum, 1993, p. 21-50.

KIM, W. C.;

MAUBORGNE, R. A.

Implementing global strategies: the role of procedural justice. Strategic Management Journal, n. 12, p. 125-143, 1991.

Procedural justice and managers' in-role and extra-role behavior: the case of the multinational. Management Science, p.499515, Apr. 1996.

Fair process: managing in the knowledge economy. Harvard Business Review, v. 4, n. 75, p. 65-75, 1997.

KONOVSKY, M. A.

Understanding procedural justice and its impacts on business organizations. Journal of Management, v. 3, n. 26, p. 489511, 2000.

KONOVSKY, M. A.;

CROPANZANO, R.

Perceived fairness of employee drug testing as a predictor of employee attitudes and job performance. Journal of Applied Psychology, n. 76, p. 698-707, 1991.

LIND, E. A.;

TYLER, T. R.

The social psychology of procedural justice. New York: Plenum, 1988.

MACKENZIE, S. B.; PODSAKOFF, P. M.; AHEARNE, M.

Some possible antecedents and consequences of in-role and extrarole salesperson performance. Journal of Marketing, n. 62, p. 87-98, 1998.

MAEL, F.;

ASHFORTH, B. E.

Alumni and their alma mater: a partial test of the reformulated model of organizational identification. Journal of Organizational Behavior, n. 13, p. 103-123, 1992.

MAIGNAN, I.;

FERRELL, O. C.; HULT, G. T. M.

Corporate citizenship: cultural antecedents and business profits. Journal of the Academy of Marketing Science, v. 4, n. 7, p. 455-469, 1999. 
MARTIN, J.;

HARDER, J. W.

Bread and roses: justice and the distribution of financial and socioemotional rewards in organizations. Social Justice Research, v. 3, n. 7, p. 241-264, 1994.

MCFARLIN, D. B.;

SWEENEY, P. D.

Distributive and procedural justice as predictors of satisfaction with personal and organizational outcomes. Academy of Management Journal, v. 3, n. 35, p. 626-637, 1992.

Cross-cultural applications of organizational justice. In: CROPANZANO, R. (Ed.). Justice in the workplace: from theory to practice. London: Lawrence Erlbaum, 2001. p. 67-95.

MCGEE, G. W.;

FORD, R. C.

Two (or more?) dimensions of organizational commitment: reexamination of the affective and continuance commitment scales. Journal of Applied Psychology, n. 72, p. 638-641, 1987.

MEDEIROS, C. A. F.

C o m p r o m e t i m e n t o organizacional, características pessoais e performance no trabalho: um estudo dos padrões de comprometimento organizacional. Trabalho apresentado no Departamento de Ciências
Administrativas, Centro de Ciências Sociais Aplicadas, Universidade Federal do Rio Grande do Norte, Natal, 1997.

MEDEIROS, C. A.;

ENDERS, W. T.

Padrões de comprometimento organizacional e suas características pessoais: como são os comprometidos e os descomprometidos com as organizações. In: ENCONTRO ANUAL DA ANPAD, 22., 1998, Foz do Iguaçu. Anais... Rio de Janeiro: ANPAD, 1998. 1 CDROM.

MEYER, J. P.;

ALLEN, N. J.

A three-component conceptualization of organization commitment. Human Resource Management Review, n. 1, p. 61-98, 1991.

MEYER, J. P.;

ALLEN, N. J.;

SMITH, C. A. Commitment to organizations and occupations: extension and test of a threecomponent conceptualization. Journal of Applied Psychology, v. 4, n. 7, p. 538-551, 1993.

MEYER, J. P. et al.

Organizational commitment and job performance: it's nature of the commitment that counts. Journal of Applied Psychology, n. 74, p. 152-156, 1989. 
MEYER, J. P.;

HERSCOVITCH, L.

Commitment in the workplace: toward a general model. Human Resource Management

Review, n. 11, p. 299-326, 2001.

MOORMAN, R. H.;

NIEHOFF, B. P.;

ORGAN, D. W.

Treating employees fairly and organizational citizenship behavior: sorting the effects of job satisfaction, organizational commitment, and procedural justice. Employee Responsibilities and Rights Journal, v. 3, n. 6, p. 209-225, 1993.

MORRISON, E. W.;

ROBINSON, S. L.

When employees feel betrayed: a model of how psychological contract violation develops. Academy of Management Review, v. 1, n. 22, p. 226-256, 1997.

MOSSHOLDER, K. W.;

BENNETT, N.;

MARTIN, C.

A multilevel analysis of procedural justice context. Journal of Organizational Behavior, n.19, p. 131-141, 1998.

MOWDAY, R. T.;

STEERS, R. M.;

PORTER, L. W.

The measurement of organizational commitment. Journal of Vocational Behavior, n. 14, p. 224-247, 1979.
NUNNALLY, J. C.

Psychometric theory. 2. ed. New York: McGraw-Hill, 1978.

O'REILLY, C.; CHATMAN, J.

Organizational commitment and psychological attachment: the effects of compliance, identification, and internalization on prosocial behavior. Journal of Applied Psychology, v. 3, n. 7, p. 492-499, 1986.

\section{PAVULS, U.}

Exploratory factor analysis of organizational commitment in Easterne Europe. Trabalho apresentado no XXVII International Congress of Psychology, Estocolmo, Suécia, 2000.

PODSAKOFF, P. M.;

MACKENZIE, S. B.;

BOMMER, W. H.

Transformational leader behaviors and substitutes for leadership as determinants of employee satisfaction, commitment, trust, and organizational citizenship behaviors. Journal of Management, v. 2, n. 22, p. 259298, 1996.

PODSAKOFF, P. M.; ORGAN, D. W.

Self-reports in organizational research: problems and prospects. Journal of Management, n. 12, p. 531-544, 1986. 
PORTER, L. W.;

STEERS, R. M.;

MOWDAY, R. T.

Organizational commitment, job satisfaction, and turnover among psychiatric technicians. Journal of Applied Psychology, v. 5, n. 59, p. 603-609, 1974.

\section{REGO, A.}

Comportamentos de cidadania organizacional: uma abordagem empírica a alguns dos seus antecedentes e conseqüências. Lisboa, 2000a. Tese (Doutorado em Gestão de Empresas) Instituto Superior de Ciências do Trabalho e da Empresa.

Justiça e cidadania nas organizações: uma abordagem sem tabus. Lisboa: Edições Sílabo, 2000b.

Justiça organizacional: desenvolvimento e validação de um instrumento de medida. Psicologia, v. 2, n. XIV, p. 285307, 2000c.

\section{Comportamentos de cidadania} nas organizações: um passo na senda da excelência? Lisboa: McGraw-Hill, 2002.

Empenhamento organizacional e ausência psicológica: afinal quantas dimensões? Revista de Administração de Empresas, 2003. No prelo.
ROCHA, A. S. C.;

BASTOS, A. V.

Comprometimento do empregado e contextos organizacionais em mudança: o caso do Banco do Brasil. Revista de Ciências Humanas, v. 5, p. 117-140, 2000.

ROUSSEAU, D. M.

Psychological contracts in organizations: understanding written and unwritten agreements. London: Sage Publications, 1995.

SCHAUBROECK, J.;

MAY, D. R.;

BROWN, F. W.

Procedural justice explanations and employee reactions to economic hardship: a field experiment. Journal of Applied Psychology, v. 3, n. 79, p. 455460, 1994.

SHORE, L. M.;

BARKSDALE, K.;

SHORE, T. H.

Managerial perceptions of employee commitment to the organization. Academy of Management Journal, v. 6, n. 38, p. 1593-1615, 1995. 
SIQUEIRA, M. M. M.

Comprometimento organizacional afetivo, calculativo e normativo: evidências acerca da validade discriminante de três medidas brasileiras. In: ENCONTRO ANUAL DA ANPAD, 25., 2001, Campinas. Anais... Rio de Janeiro: ANPAD, 2001. 1 CDROM.

SKARLICKI, D. P.;

FOLGER, R.

Retaliation in the workplace: the roles of distributive, procedural, and interactional justice. Journal of Applied Psychology, v. 3, n. 82, p. 434-443, 1997.

SLOCOMBE, T. E.;

BLUEDORN, A. C.

Organizational behavior implications of the congruence between preferred polychronicity and experiential work-unit polychronicity. Journal of Organizational Behavior, n. 20, p. 75-99, 1999.

SWAILES, S.

Organizational commitment: searching for the Holy Grail of HRM. Comportamento Organizacional e Gestão, v. 2, n. 6, p. 199-212, 2000.

SWEENEY, P. D.;

MCFARLIN, D. B.

Workers' evaluations of the 'ends' and the 'means': an examination of four models of distributive and procedural justice. Organizational Behavior and Human Decision Processes, n. 55, p. 23-40, 1993.

Process and outcome: gender differences in the assessment of justice. Journal of Organizational Behavior, n. 18, p. 83-98, 1997.

THIBAUT, J.;

WALKER, J.

Procedural justice: a psychological analysis. London: Lawrence Erlbaum, 1975.

TYLER, T. R.;

BIES, R. J.

Beyond formal procedures: the interpersonal context of procedural justice. In: CARROLL, J. S. (Ed.). Applied social psychology in business settings. London: Lawrence Erlbaum, 1990. p. 7798.

TYLER, T.;

DEGOEY, P.;

SMITH, $\mathrm{H}$.

Understanding why the justice of group procedures matters: a test of the psychological dynamics of the group-value model. Journal of Personality and Social Psychology, v. 5, n. 70, p. 913930, 1996. 
VAN KNIPPENBERG, D.;

VAN SCHIE, E. C. M.

Foci and correlates of organizational identification. Journal of Occupational and Organizational Psychology, n. 73, p. 137-147, 2000.
YOUSEF, D. A.

Organizational commitment: a mediator of the relationships of leadership behavior with job satisfaction and performance in a non-western country. Journal of Managerial Psychology, v. 1, n. 15, p. 6-28, 2000. 